\title{
The impact of laser-assisted hatching on the outcome of frozen human embryo transfer cycles
}

\author{
Katalin Kanyo ${ }^{2}$, Jozsef Zeke ${ }^{2}$, Rita Kriston ${ }^{2}, Z_{\text {Zoltan Szücs }}^{2}$, Sandor Cseh ${ }^{1,2}$, Bence Somoskoi ${ }^{3}$ \\ and Janos Konc ${ }^{2}$ \\ Infertility and IVF Center of Buda, Szent Janos Hospital, Budapest, Hungary; and Szent Istvan University Faculty of \\ Veterinary Science, Budapest, Hungary
}

Date submitted: 10.03.2015. Date revised: 08.10.2015. Date accepted: 14.01.2016

\section{Summary}

Biochemical modifications of zona pellucida $(\mathrm{ZP})$ result in zona hardening. Zona hardening $(\mathrm{ZH})$ is induced by several factors such as advancing maternal age, in vitro culture conditions and cryopreservation and adversely effects implantation. The objective of the clinical study was to determine whether or not laser-assisted hatching (LAH) applied on day 3 frozen embryos improves the outcome of frozen embryo transfer (FET) cycles in patients with recurrent implantation failure and/or advanced female age. In total, 413 patients of different ages with recurrent implantation failure (maximum three cycles) were involved into the study. Patients were allocated randomly into LAH and control groups. On the day of FET, after thawing and just before FET, the ZP was thinned using a laser system. In the control group no treatment was applied on frozen embryo before transfer. The main outcome measures were clinical pregnancy rate. Overall, the results indicate a tendency that LAH increased $(P=0.08)$ clinical pregnancy. However, for patients older than 37 years, LAH increased pregnancy rates significantly $(P=0.03)$. In the LAH and control groups, the age of patients and the number of transferred embryos influenced pregnancy rates $(P=0.01)$. For patients older than 37 years, no effect of number of transferred embryos was detected $(P=0.14)$. The incidence of multiple pregnancies also increased in the LAH group $(P=0.01)$. In conclusion, in older woman, to overcome the negative effect of zona hardening, LAH could be performed on frozen embryos as a routine strategy before FET in frozen cycles in order to increase the possibility of pregnancy formation.

Keywords: Age of patients, Clinical outcome of laser-assisted hatching, Frozen embryo transfer cycle, Laser-assisted hatching, Slow freezing of embryos

\section{Introduction}

The zona pellucida ( $\mathrm{ZP})$ is an envelope that contains a species-dependent sperm receptor, it triggers the acrosome reaction and in the frame of a physiological process after fertilization undergoes biochemical modifications called zona hardening that prevent polyspermy (Cohen, 2007). The ZP ruptures at the blastocyst stage in a process called hatching and

\footnotetext{
${ }^{1}$ All correspondence to: Sandor Cseh. 1078 Budapest, Istvan u. 2. Hungary. Tel: +36 30210 1332. Fax: +3614784207. E-mail: cseh.sandor@aotk.szie.hu

${ }^{2}$ Infertility and IVF Center of Buda, Szent Janos Hospital, Budapest 1125, Hungary.

${ }^{3}$ Szent Istvan University Faculty of Veterinary Science, Budapest 1078, Hungary.
}

this event allows embryo implantation. Mechanical expansion and contraction/elasticity of the blastocyst and, furthermore, thinning of the ZP are all involved in hatching and are essential for this process (Cohen, 2007). Both elasticity / zona hardening and ZP thinning can be influenced adversely by advancing maternal age and in vitro culture conditions (Cohen et al., 1992; Huisman et al., 2000). Data indicate that zona hardening may be initiated by cryopreservation as well. (Cohen et al., 1999; Elhussieny et al., 2013).

Abnormalities in the hatching have been suggested as a possible explanation for the low implantation rates observed in some patients, in advanced maternal age and in frozen-thawed IVF/ICSI-ET cycles (Cohen, 2007). Assisted hatching (AH) procedures are based on creating an artificial opening or thinning $\mathrm{ZP}$, which 
is believed to support hatching and implantation of embryos that are otherwise unable to escape from the ZP (Cohen et al., 1992). Yet, the effectiveness of AH to improve the outcome of fresh and frozen IVF/ICSI-ET cycles remains controversial ( $\mathrm{Ng}$ et al., 2005; Ge et al., 2008; Lan et al., 2009; Carney et al., 2012; Razi et al., 2013; Wan et al., 2014).

Our clinical study focused on the use of a laser zona thinning (laser-assisted hatching; LAH) method on day 3 cryopreserved embryos with the hypothesis that it may be a useful tool for improving live birth rates especially in advanced female age in frozenthawed ICSI-ET cycles.

\section{Materials and methods}

\section{Patient selection, controlled ovarian stimulation, oocyte recovery}

Laser-assisted hatching procedure has been used for 11 years at our IVF Centre in the clinical work (Kanyo \& Konc, 2003). All patients (age: 21-43 years) received written information/coverage about the study and were asked to agree to the conditions of the study and to take part in it. They signed a written consent. Patients were allocated randomly into the LAH+ and LAH- groups based on the last number/digit of their registration number given to them by the administrator at the reception desk during their first visit to our centre. The embryologists were not informed about the deployment of the patients.

In total, 413 (203 with $\mathrm{LAH}+$ and 210 with no LAH-) undertook thaw-transfer cycles within a 12month period were analysed in the Infertility and IVF unit of a Medical Centre. All patients met the following criteria: (1) maximum three previous IVFembryo transfer (IVF-ET); and (2) having reached ET process. Indications for IVF-ET were: male factor $(40 \%)$, tubal factor $(34 \%)$, endometriosis $(15 \%)$ and unexplained infertility (11\%). The reader is referred to our previous publications for stimulation protocols, oocyte recovery and ET techniques (Kanyo \& Konc, 2003; Konc et al., 2005, 2010; Zeke et al., 2012).

On the day of embryo thawing and ET, the patients - according to the random allocation done during their first visit to the center when they signed up for the IVF program - were grouped into two groups: the LAH+ group and the LAH - group. The aim was to determine if LAH could increase the pregnancy rate in frozen ET cycles.

\section{Fresh embryo culture}

The G series sequential culture system (VitroLife, Gothenburg, Sweden) was used for in vitro culture of embryos. Embryo culture was performed as described previously (Konc et al., 2005, 2010; Zeke et al., 2012).

\section{Cryopreservation of embryos}

Embryos were frozen on day 3 after in vitro fertilization with ICSI in phosphate-buffered saline solution (PBS) supplemented with $1.5 \mathrm{M}$ 1,2-propanediol $(\mathrm{PrOH}), 25 \mathrm{mg} / \mathrm{ml}$ human serum albumin (HSA) and $0.1 \mathrm{M}$ sucrose (S) (VitroLife, Gothenburg, Sweden). For freezing, a conventional slow freezing protocol was used with a PLANER III Kryo 10 cell freezer (Planer Products Ltd, Sunbury-on-Thames, UK). After seeding at $-7^{\circ} \mathrm{C}$, the embryos were slowly cooled $\left(0.3^{\circ} \mathrm{C} / \mathrm{min}\right)$ to $-30^{\circ} \mathrm{C}$, then the temperature was decreased rapidly $\left(-10^{\circ} \mathrm{C} / \mathrm{min}\right)$ to $-80^{\circ} \mathrm{C}$ before plunging the embryos into liquid nitrogen $\left(\mathrm{LN}_{2}\right)$.

Only good quality day 3 embryos were frozen. Good quality embryos were defined as those having regular blastomeres, $<20 \%$ fragments and no multinucleated blastomeres and those containing at least seven cells on day 3 in both LAH and control groups.

\section{Thawing protocol}

During embryo thawing first air thaw was applied for $20 \mathrm{~s}$ followed by immersion into $30^{\circ} \mathrm{C}$ water bath for $20 \mathrm{~s}$. After thawing, $\mathrm{PrOH}$ was removed from the cells in three steps with solutions containing $\mathrm{PrOH}$ in decreasing concentrations and $0.2 \mathrm{M} \mathrm{S}$ (VitroLife, Gothenburg, Sweden). After dilution out of the cryoprotectant, embryos were cultured in vitro for 1-1.5 $\mathrm{h}$ prior to final quality assessment and ET. Cryopreserved embryos were considered to have survived if $>50 \%$ of the blastomere were intact. LAH was used just before ET on embryos in the LAH group $(n=276)$. In the control group, no LAH was used on embryos prior to ET. A maximum of 2-3 embryos was transferred per patient.

\section{Laser-assisted hatching (LAH) of embryos}

Embryo thawing was performed in the morning of ET, and LAH was carried out just before ET. LAH was performed only on embryos that survived following thawing (see before). Assisted hatching of human embryos was performed with a 'non-contact' 670 $\mathrm{nm}, 1.48 \mu \mathrm{m}$, infra-red diode laser (Fertilase', MTM, Germany) (Table 1). A $1.48 \mu \mathrm{m}$ continuous wave laser beam, which was collimated with a $1 \mathrm{~mW}$ visible 670 nanometer diode laser beam were fed into an inverted microscope through several mirrors and focused by a $\times 45$ microscope objective. This led to a measured spot size of $1-3 \mu \mathrm{m}$ in diameter. This spot was magnified and observed on an external monitor. The infra-red diode laser beam, focused through the microscope objective, is activated by using a foot pedal and causes 
Table 1 Patient characteristics and embryo transfer data in the laser and no-laser groups

\begin{tabular}{lccc}
\hline & LAH & No LAH & $P$-value \\
\hline Number of patients & 203 & 210 \\
Mean female age (years) & $33.35 \pm 3.3$ & $33.04 \pm 3.1$ \\
Duration of infertility (years) & 7.1 & 6.8 & \\
Mean number of previous failed ART cycles & 1.9 & 1.7 & \\
Mean BMI & 23.71 & 23.45 & NS \\
Number of pregnant patients (\%) & $59(33.3)$ & $51(27.4)$ & NS \\
Number of abortions including biochemical pregnancies (\%) & $34(16.74)$ & $33(15.71)$ & \\
\hline
\end{tabular}

a trench or a tunnel of the ZP. The drilling effect is due to a highly localized disruption of ZP glycoprotein matrix, which is stimulated by heat without causing ionization. The tangential laser irradiation results in a trench or tunnel-like hole (the diameter of the hole is $4.5-20 \mu \mathrm{m})$, at an optimal laser power setting of $47 \mathrm{~mW}$.

The ZP was exposed for $10-15 \mathrm{~ms}$ to the laser beam. The diameter of the drilled holes varied between 5$10 \mu \mathrm{m}$ depending on the irradiation time and the temperature of the surrounding medium. One or a maximum of two shots were performed. The embryos were transferred within 20 min (Kanyo \& Konc, 2003).

\section{Endometrial preparation and transfer of frozen-thawed embryos}

First, patients were downregulated starting in the midluteal phase of the preceding menstrual cycle. Subsequently, endometrial development was supported by administration of $17 \beta$-estradiol valerate (Estrofen, tabl. $2 \mathrm{mg}$, Novo Nordisk) in increasing doses (2$6 \mathrm{mg} /$ day) to mimic the natural cycle which was commenced orally on day 2 of the menstrual cycle and continued until endometrial thickness reached $>8 \mathrm{~mm}$. Once endometrial development was confirmed by ultrasound, progesterone was administered (600 mg/day; Utrogestan, Lab. Besins Internat S.A.) to support the luteal phase.

Embryo transfer was carried out on day 3 and prior to ET, LAH was performed on each embryo (MTG, Fertilase, Hamburg, Germany). Pregnancy was defined as a spontaneous rise in a $\beta$-hCG concentration at least 10 days post-transfer. Clinical pregnancy implied the presence of an intrauterine gestational sac and fetal heart beat on an ultrasound performed at 7 weeks of gestation (Zeke et al., 2012). In case of spontaneous abortion, the patient lost her biochemical or clinical pregnancy.

\section{Statistical analysis}

Data were analyzed by logistic regression using SPLUS 2000. The outcome variable was the clinical pregnancy and the predictor was preparation of the embryos (LAH+ and LAH-) to ET. A comparison was also made between age, body mass index (BMI) and the number of transferred embryos and clinical pregnancy rate. Patients whose clinical pregnancy ended with a birth were considered as pregnant. Biochemical pregnancies were excluded from this group. Power calculations were also made and we reported a 95\% confidence interval $(95 \% \mathrm{CI})$ for the odds ratios (OR) associated with the factors included in the model.

\section{Results}

In the frame of this study, the results of 413 frozenthawed ET cycle were evaluated. Out of the 413 ET cycles in 203, LAH was applied on the embryos just prior to transfer. In the control group, none of the transferred embryos was treated with LAH. The mean female age (at the time of the fresh ET), duration of infertility, mean number of previous failed ART cycles and, main BMI, number of biochemical or clinical pregnancies, number of spontaneous abortions were similar in the LAH+ and LAH - groups (Table 1).

Likewise, cryo-survival was also similar in the two groups (LAH+: $92.0 \%$ vs. LAH-: 91.4\%). Comparing $\mathrm{LAH}+$ and LAH- groups in the overall patient population, we could detect that LAH supports ( $33.3 \%$ vs. $27.4 \%$, respectively, $P=0.08$; Fig. 1 ) the formation of clinical pregnancy. The statistical analyses, however, show only a trend but the results were not significantly different. When we compared the pregnancy rates of the LAH+ and LAH- groups based on the age of the patients, we found that in patients who were over $37(\geq 38)$ years of age, LAH significantly increased the pregnancy rates $(18.36 \%$ vs. $11.36 \%$, respectively, $P=0.03$ ) (Table 2). In patients under the age of 37 , 'only' a non-significant increase (trend/tendency) in the pregnancy rate was detected in the LAH+ group $(28.4 \%$ vs. $23.6 \%$, respectively). In younger patients ( $<38$ years), however, our data clearly show that patients' age had a positive supportive effect 
Table 2 Patient characteristics and embryo transfer data in the laser and no-laser groups of patients with above 37 years of age

\begin{tabular}{lccc}
\hline & LAH, $>37$ & No LAH, $>37$ & $P$-value \\
\hline Number of patients & 49 & 44 & \\
Duration of infertility (years) & 7.4 & 7.2 & \\
Mean number of previous failed ART cycles & 2.1 & 1.9 & \\
Mean BMI & 23.91 & 23.65 & \\
Number of pregnant patients (\%) & $9(18.36)$ & $5(11.36)$ & $P=0.03$ \\
Number of abortion including biochemical pregnancies (\%) & $7(14.28)$ & $7(15.90)$ & NS \\
\hline
\end{tabular}

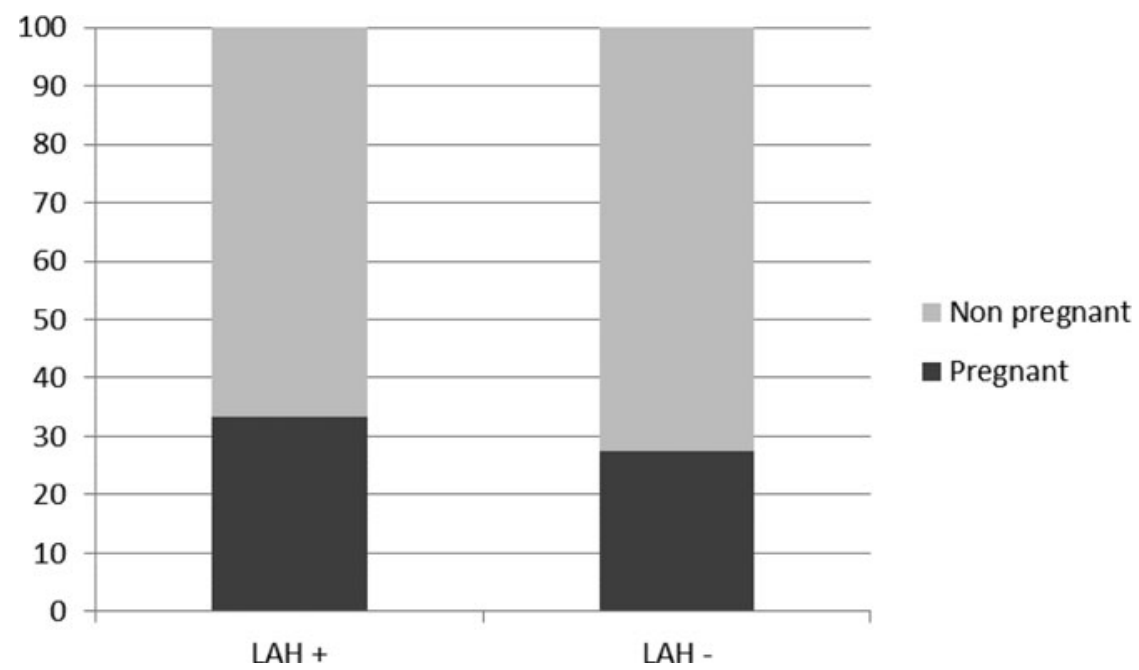

Figure 1 The overall pregnancy rates obtained in the LAH+ and LAH- groups.

on clinical pregnancy establishment, and younger patients got pregnant more easily $(P=0.003)$ compared with older patients.

Our data also indicate that the number of transferred embryos has a strong positive influence on clinical pregnancy (two embryos: 10/49, 20\%; three embryos: 8/29, 27\%; four embryos: 1/3,33\%). But for patients above the age of 37 , we were unable to prove statistically the supportive effect of the number of transferred embryos. Likewise, no effect of BMI on clinical pregnancy rate was observed. However, not only the clinical pregnancy rate, but the incidence of multiple pregnancies, was increased in the $\mathrm{LAH}+$ group $(5.8 \%$ vs. $3.3 \%$ for $\mathrm{LAH}+$ and $\mathrm{LAH}-$, respectively, $P<0.01$ ).

\section{Discussion}

Prior to implantation, the blastocyst has to hatch from the ZP. The premise behind AH is based on the assumption that a modification of the human ZP might promote hatching and implantation of embryos that are otherwise unable to escape from the intact ZP (Cohen et al., 1992; Cohen, 2007). The modification could be carried out either by its elimination, by drilling a hole, by thinning, or by altering its stability. The use of different $\mathrm{AH}$ procedures is based on the assumption that creating an artificial hole/opening or thinning the $\mathrm{ZP}$ might assist the hatching process of embryos.

$\mathrm{AH}$ supports in vivo hatching of the blastocyst and therefore may improve the efficacy of pregnancy rates. Assisted hatching was reported to increase the outcome of IVF-ET cycles in groups of patients with poor prognosis and/or poor embryonic morphology, with elevated FSH levels, with embryos having thicker $\mathrm{ZP}$, who were older and with a history of multiple IVF failures. Thick ZP may be associated with advanced female age and poor embryo scores, and furthermore with cryopreservation (Cohen et al., 1992; Cohen, 2007; Valojerdi et al., 2008; Carney et al., 2012; Wan et al., 2014).

There is a sharp contradiction in the results and observations of different studies dealing with $\mathrm{AH}$. Because of the discrepancy in the outcomes between the different studies, results obtained from them should be interpreted with caution ( $\mathrm{Ng}$ et al., 2005; Ge et al., 2008; Valojerdi et al., 2008; Feng et al., 2009; Lan et al., 2009; Carney et al., 2012; Wan et al., 2014). 
Comparing the pregnancy rates of fresh IVF cycles with poor prognosis patients (prior IVF failures) after using different techniques of $\mathrm{AH}$, no difference was found in the efficacy of these and all of the techniques (laser, chemical or microsurgical) increased pregnancy rates (Feng et al., 2009). However, Baruffi et al. (2000) in a prospective randomized study found no difference in pregnancy rates in a population aged $<37$ years and with no previous implantation failure (Baruffi et al., 2000). Moreover, Frydman et al. (2006) found that LAH did not improve the IVF-embryo transfer outcome in woman aged $>37$ years (Frydman et al., 2006).

Cryopreservation may induce zona hardening similarly to in vitro culture and advanced female age (Larman et al., 2006). Assisted hatching may overcome this problem and increase implantation and pregnancy rates in frozen-thawed ET cycles. Similarly to the fresh IVF cycles, the results obtained after AH in frozen-ET cycles are very conflicting (Carney et al., 2012).

Assisted hatching with chemical zona drilling in frozen-thawed cycles has been shown to increase pregnancy rates in retrospective studies (Cohen et al., 1999). However, in other studies using similar techniques no advantage of the procedure could be detected in cryopreserved ICSI-ET cycles (Edirisinghe et al., 1999). In a recently published randomized study, $\mathrm{Ng}$ et al. (2005) failed to show any beneficial effect of LAH on pregnancy rate following the transfer of cryopreserved embryos ( $\mathrm{Ng}$ et al., 2005). Primi et al. (2004) were also unable to show any benefit of $\mathrm{AH}$ in frozen-thawed ET cycles (Primi et al., 2004). In the $\mathrm{Ng}$ et al. (2005) study, patients of advanced age also did not benefit from LAH, they were able to detect only a trend towards increased pregnancy rates $(\mathrm{Ng}$ et al., 2005). Their observation is in contrast with ours, as we found that LAH increased significantly the pregnancy rate in patients over the age of 37 . In younger patients, less than 37 years old, we could see only a trend of increased pregnancy rate in the LAH+ group. LAH improved the pregnancy rates in patients with frozen-thawed embryos, but they observed no effect in patients with advanced female age.

Our results obtained in frozen-thawed ICSI-ET cycles show that LAH increases pregnancy rates. The patient population was quite similar in the two groups, likewise the embryo characteristics and the applied cryopreservation and embryo culture procedures, thus precluding any bias that may have influenced our results. Similarly to our results, other studies also found that LAH significantly increased pregnancy rates in cryopreserved cycles with slowly frozen and vitrified embryos compared with fresh cycles (Ebner et al., 2005; Zhang et al., 2009).

The possible explanation of the less successful application of LAH could be that the size of the $\mathrm{ZP}$ thinning may have an effect on the results. Zhang et al. (2009) compared the effect of the size of the ZP thinning $(40 \mu \mathrm{m}$ vs. $80 \mu \mathrm{m})$ made by the laser on the outcome of frozen-thawed ET cycles. They found that the size of the thinning by the laser may influence the implantation and pregnancy rates (they got increased implantation and pregnancy when $80 \mu \mathrm{m}$ thinning was made) following frozen-thawed cleaved embryo transfer (Zhang et al., 2009). The data from Hiraoka et al.'s study (2009) show that in vitrified-warmed embryo transfers the size of the $\mathrm{ZP}$ thinning area by LAH impacts the clinical pregnancy rate and that one-half ZP thinning significantly increases the results compared with one-quarter ZP thinning (Hiraoka et al., 2009).

\section{Conclusion}

In summary, the data from our study show that LAH may be useful for improving the pregnancy rates using frozen day 3 ET in recurrent pregnancy failure patients over 37 years. According to our results, LAH increased pregnancy rates in woman who were aged over 37 years and taking part in FET. In patients younger than 37 years old we could detect only a trend towards increased pregnancy rates in the $\mathrm{LAH}+$ group compared with the LAH- group. The number of transferred embryos has a strong positive effect on pregnancy formation, but only in patients younger than 37 years of age. In people over 37 years old this positive effect of embryo number on pregnancy could not be detected. The incidence of multiple pregnancies was increased in the LAH+ group. Considering our data and to overcome the negative effect of zona hardening, which is very typical in older recurrent pregnancy failure woman, plus the fact that cryopreservation makes the situation more difficult, we suggest that LAH could be performed on frozen-thawed embryos as a routine strategy in FET cycles before they are transferred.

\section{References}

Baruffi, R., Mauri, A., Petersen, C., Ferreira, R., Coelho, J. \& Franco, J. (2000). Zona thinning with noncontact diode laser in patients aged $<37$ years with no previous failure of implantation: a prospective randomized study. J. Assist. Reprod. Genet. 17, 557-60.

Carney, S.K., Das, S., Blake, D., Farquhar, C., Seif, M.M. \& Nelson, L. (2012). Assisted hatching on assisted conception (in vitro fertilisation) (IVF) and intracytoplasmic sperm injection (ICSI). Cochrane Database Syst. Rev. 12, CD001894.

Cohen, J. (2007). Manipulating embryo development. In Human Preimplantation Embryo Selection (eds K. Elder \& J. Cohen). Abingdon UK: Informa Healthcare, Informa UK Ltd. 
Cohen, J., Alikani, M., Trowbridge, J. \& Rosenwaks, Z. (1992). Implantation enhancement by selective assisted hatching using zona drilling of human embryos with poor prognosis. Hum. Reprod. 7, 685-91.

Cohen, J., Lindheim, S. \& Sauer, M. (1999). Assisted hatching causes beneficial effects on the outcome of subsequent frozen embryos transfers of donor oocyte cycle. Fertil. Steril. 72 (Suppl. 1), S5.

Ebner, T., Moser, M. \& Tews, G. (2005). Possible applications of a non-contact $1.48 \mu \mathrm{m}$ wavelength diode laser in assisted reproduction technologies. Hum. Reprod. Update 11, 425-35.

Elhussieny, A., El Mandoub, M., Hanafi, S., Mansour, G.M. \& El-Kotb, A. (2013). Effect of laser assisted hatching on outcome of assisted reproductive technology. Open J. Obstet. Gynecol. 3, 18-23.

Edirisinghe, W., Ahnonkitpanit, V., Promviengchai, S., Suwajanakom, S., Pruksananonda, K., Chinpilas, V. \& Virutamasen, P. (1999). A study failing to determine significant benefits from assisted hatching: patients selected for advanced age, zona thickness of embryos, and previous failed attempts. J. Assist. Reprod. Genet. 16, 294 301.

Feng, H.L., Hershlag, A., Scholl, G.M. \& Cohen, M.A. (2009). A retrospective study comparing three different assisted hatching techniques. Fertil. Steril. 91, 1323-5.

Frydman, N., Madoux, S., Hesters, L., Duvernoy, C., Feyereisen, E., Le Du, A., Tachdijian, G., Frydman, R. \& Fanchin, R. (2006). A randomized double-blind controlled study on the efficacy of laser zona pellucida thinning on libe birth rates in cases of advanced female age. Hum. Reprod. 21, 2131-5.

Ge, H.S., Zhou, W., Zhang, W. \& Lin, J.J. (2008). Impact of assisted hatching on fresh and frozen-thawed embryo transfer cycles: a prospective, randomized study. Reprod. Biomed. Online 16, 589-96.

Hiraoka, K., Hiraoka, K., Horiuchi, T., Kusuda, T., Okano, S., Kinutani, M. \& Kinutani, K. (2009). Impact of the size of the zona pellucida thinning area on vitrifiedwarmed cleavage-stage embryo transfers: a prospective, randomized study. J. Assist. Reprod. Genet. 26, 515-21.

Huisman, G.J., Fauser, B.C., Eijkemans, M.J. \& Pieters, M.H. (2000). Implantation rates after in vitro fertilization and transfer of a maximum of two embryos that have undergone three to five days in culture. Fertil. Steril. 73, 117-22.

Kanyo, K. \& Konc, J. (2003). A follow-up study of children born after diode laser assisted hatching. Eur. J. Obstet. Gynecol. Reprod. Biol. 110, 176-80.

Konc, J., Kanyo, K. \& Cseh, S. (2005). Clinical experiences of ICSI-ET thawing cycles with embryos cryopreserved at different developmental stages. J. Assist. Reprod. Genet. 22, 185-90.

Konc, J., Kanyo, K., Varga, E., Kriston, R. \& Cseh, S. (2010). The effect of cycle regimen used for endometrium preparation on the outcome of day 3 frozen embryo transfer cycle. Fertil. Steril. 94, 767-8.

Lan, K.C., Huang, F.J., Lin, Y.C., Kung, F.T. \& Chang, S.Y. (2009). Zona-free versus laser zona-assisted hatching blastocyst transfer: a comparision of outcomes. Fertil. Steril. 91, 1959-62.

Larman, M.G., Sheehan, C.B. \& Gardner, D.K. (2006). Calcium-free vitrification reduces cryoprotectant-induced zona pellucida hardening and increases fertilization rate in mouse oocytes. Reproduction 131, 53-61.

Ng, E., Naveed, F., Lau, R., Yeung, W.S., Chan, C.C., Tang, O.C. \& Ho, P.C. (2005). A randomized double blind controlled study of the efficacy of laser assisted hatching on implantation and pregnancy rates of frozen-thawed embryo transfer at the cleavage stage. Hum. Reprod. 20, 979-85.

Primi, M., Senn, A., Montag, M., Van der Ven, H., Mandelbaum, J., Veiga, A., Barri, P. \& Germond, M. (2004). A European multicentre prospective randomized study to assess the use of assisted hatching with a diode laser and the benefit of an immunosuppressive/antibiotic treatment in different patient populations. Hum. Reprod. 19, 232533.

Razi, M.H., Halvaei, I. \& Razi, Y. (2013). Laser assisted hatching does not improve live birth rate in patients undergoing their first ICSI cycles. Iran J. Reprod. Med. 11, 1021-6.

Valojerdi, M.R., Eftekhari, Y.P., Karimian, L. \& Ashtiani, S.K. (2008). Effect of laser zona pellucida opening on clinical outcome of assisted reproduction technology in patients with advanced female age, recurrent implantation failure, or frozen-thawed embryos. Fertil. Steril. 90, 84-91.

Wan, C.Y., Song, C., Diao, L.H., Li, G.G., Bao, Z.J., Hu, X.D., Zhang, H.Z. \& Zeng, Y. (2014). Laser-assisted hatching improves clinical outcomes of vitrified-warmed blastocysts developed from low-grade cleavage-stage embryos: a prospective randomized study. Reprod. Biomed. Online 28, 582-9.

Zeke, J., Konc, J., Kanyo, K., Kriston, R. \& Cseh, S. (2012). Birth and clinical pregnancy from fresh and frozen oocytes fertilized with cryopreserved testicular spermatozoa. Syst. Biol. Reprod. Med. 58, 165-7.

Zhang, X.J., Yang, Y.Z., Lv, Q., Min, L.H., Li, X.L. \& Bai, P. (2009). Effect of the size of zona pellucida thinning by Laser assisted hatching on clinical outcome of human frozen-thawed embryo transfers. Cryo Lett. 30, 455-61. 